\title{
Outlook for intracerebral haemorrhage after a MISTIE spell
}

\author{
David J Werring PhD FRCP
}

Stroke Research Centre, Department of Brain Repair and Rehabilitation, UCL Institute of Neurology, National Hospital for Neurology and Neurosurgery, Queen Square, London WC1N 3BG

Corresponding author: Prof Werring d.werring@ucl.ac.uk

(747 words) 
Accounting for about $15 \%$ of all strokes, with one-month mortality around $40 \%{ }^{1}$, spontaneous, nontraumatic acute intracerebral haemorrhage (ICH) causes a substantial global disease burden. Although stroke unit care ${ }^{2}$ and acute blood pressure lowering improve outcome ${ }^{3}$, there is an urgent need for more effective acute treatments. Craniotomy and surgical evacuation aims to reduce or remove the haematoma, thus reducing mass effect and direct or secondary tissue injury, yet has not shown benefit in large pragmatic randomized controlled trials ${ }^{4}$. Less invasive strategies aim to achieve haematoma removal or reduction, with less associated brain tissue injury, and show promising outcomes in small studies ${ }^{5}$.

The minimally invasive surgery plus recombinant tissue plasminogen activator in intracerebral haemorrhage evacuation (MISTIE) trial assessed whether minimally invasive surgery (MIS) and rt-PA is safe and reduces clot burden, and obtained preliminary functional outcome data. All patients were managed in high-level care, with an intervention of a small craniotomy, image-guided catheter aspiration, thrombolysis with rt-PA, irrigation and drainage over several days. An initial rt-PA dose-finding stage led to stage two of the trial, in which 96 patients with spontaneous ICH $\geq 20 \mathrm{ml}$, "clinically stable" after 6 hours (clot expansion $<5 \mathrm{ml}$ and no active bleeding on $\mathrm{CT}$ ) were randomized to standard medical guideline-based care or MIS and rt-PA thrombolysis plus guideline-based care. The primary outcome was "good outcome" on the adjusted dichotomized modified Rankin Scale (mRS) (0-3 vs 4-6) at 180 days. Primary safety outcomes were mortality, symptomatic bleeding, brain infections, and withdrawal of care. The population included $67 \%$ men, average age 61 years, with $66 \%$ basal ganglia and $34 \%$ lobar haematoma 
locations. The mean entry ICH size was 46ml, and median Glasgow Coma Scale 10. Safety outcomes did not differ between groups. At 180 days, the proportions with good recovery (mRS $0-3$ ) were $33 \%$ in the MIS and rt-PA arm and $21 \%$ in the comparator medical arm. After adjustment for important potential imbalances in baseline severity (NIHSS, GCS, ICH volume and IVH volume) the absolute difference in the proportion achieving a favourable outcome was 0.162 [95\%CI: 0.003, 0.323; $\mathrm{p}=0.05]$. Asymptomatic and symptomatic bleeding were both more common in the MIS group ( $22 \%$ vs. $7 \%, p=0.05$; and $9.3 \%$ vs. $2.4 \%, p=0.226$, respectively).

How should clinicians interpret these results? The headlines are that MIS and rt-PA does not increase mortality, has an apparent advantage of better functional outcome at 180 days (albeit only marginally statistically significant), but causes increased bleeding (both asymptomatic and symptomatic). Nevertheless the trial provides important new randomized evidence that a standardized minimally invasive neurosurgical intervention might improve outcome after ICH. The method is theoretically attractive in minimizing cortical trauma and excision, and in reducing clot volume to reduce both mechanical and secondary inflammatory or toxic injury. However, some aspects merit careful consideration. First, of 4103 patients screened, only 96 (less than 3\%) were randomized, suggesting generalizability only to a minority of patients with $\mathrm{ICH}$, although participants with deep and lobar ICH were included. Second, the intervention is complex, requiring adherence to a lengthy study intervention protocol with varied success measures in the trial, and most likely even greater variation if translated to real-world practice. Third, although apparently not increasing mortality, the study confirms that using rt-PA increases the risk 
of bleeding, albeit not at the expense of net long-term functional benefit - analogous to the accepted early ICH risk and improved long-term functional outcome associated with rt-PA in acute ischaemic stroke 6 . Finally, this small trial was not powered to show efficacy, leading to a wide range of estimated treatment effects.

It remains uncertain to what extent the apparent benefit of MIS is due to direct clot volume reduction through aspiration (limiting mass effect), or the use of rt-PA to continue removing blood products from the hematoma cavity (potentially reducing inflammation and other toxic secondary injury). Other techniques including clot endoscopic aspiration under direct guidance (without rt-PA) might therefore be of interest ${ }^{7}$. Although exploratory analyses suggest that better clot removal is associated with improved outcome, proving this hypothesis requires further trials. Thus, although MISTIE is a promising step forward, much larger trials in $\mathrm{ICH}$ patients recruited from both stroke and neurosurgical units are essential, to ensure that the results are not due to chance and can be replicated in current practice; until then the data are unlikely to directly influence current neurosurgical approaches. Nevertheless, if MISTIE and other minimally invasive techniques can be proven to produce meaningful functional benefit in patients with $\mathrm{ICH}$, this could herald a new era in treating this most devastating of neurological emergencies.

\section{References}


1. van Asch CJ, Luitse MJ, Rinkel GJ, van der Tweel I, Algra A, Klijn CJ. Incidence, case fatality, and functional outcome of intracerebral haemorrhage over time, according to age, sex, and ethnic origin: a systematic review and meta-analysis. Lancet neurology 2010; 9(2): $167-76$.

2. Langhorne P, Fearon P, Ronning OM, et al. Stroke unit care benefits patients with intracerebral hemorrhage: systematic review and meta-analysis. Stroke; a journal of cerebral circulation 2013; 44(11): 3044-9.

3. Anderson CS, Heeley E, Huang Y, et al. Rapid blood-pressure lowering in patients with acute intracerebral hemorrhage. The New England journal of medicine 2013; 368(25): 2355-65.

4. Mendelow AD, Gregson BA, Rowan EN, et al. Early surgery versus initial conservative treatment in patients with spontaneous supratentorial lobar intracerebral haematomas (STICH II): a randomised trial. Lancet 2013; 382(9890): 397-408.

5. Zhou X, Chen J, Li Q, et al. Minimally invasive surgery for spontaneous supratentorial intracerebral hemorrhage: a meta-analysis of randomized controlled trials. Stroke; a journal of cerebral circulation 2012; 43(11): 2923-30.

6. Emberson J, Lees KR, Lyden P, et al. Effect of treatment delay, age, and stroke severity on the effects of intravenous thrombolysis with alteplase for acute ischaemic stroke: a meta-analysis of individual patient data from randomised trials. Lancet 2014.

7. Auer LM, Deinsberger W, Niederkorn K, et al. Endoscopic surgery versus medical treatment for spontaneous intracerebral hematoma: a randomized study. Journal of neurosurgery 1989; 70(4): 530-5. 
